\title{
MEASUREMENTS OF COLLISIONAL RATE COEFFICIENTS \\ IN LABORATORY PLASMAS
}

\author{
H. -J. K UNZE \\ Dept. of Physics and Astronomy, University of Maryland, College Park, Md., U.S.A.
}

\begin{abstract}
Line radiation emitted by highly ionized atoms embedded in hot laboratory plasmas can be utilized to obtain collisional rate coefficients for excitation and ionization. After a discussion of the principles underlying these measurements, the plasma device mostly used is explained briefly as are the various experimental techniques. All experimental results obtained so far are finally discussed and compared with theoretical calculations where possible.
\end{abstract}

\section{Introduction}

Hot plasmas nowadays available in various laboratory devices offer a unique opportunity of obtaining collisional rate coefficients for highly ionized atoms. Standard cross-beam techniques usually fail here, since the cross-sections not only decrease rapidly with increasing charge, but since it is also still rather difficult to obtain suitable high-current ion beams. The general principle of the measurements is the following: the atoms of interest are introduced into a well-diagnosed plasma, and the time history, the absolute intensity and the width of properly chosen lines emitted by the desired ions are interpreted then in terms of proper rate coefficients. One could consider these measurements somehow as inferior, since they do not yield the cross-sections and their detailed structure; however, for most practical applications in the laboratory as well as for the analysis of astrophysical plasmas, (Gabriel and Jordan, 1972; Griem, 1964; Elton, 1970; McWhirter, 1965), it is indeed the rate coefficient $\langle\sigma v\rangle$ which is needed, i.e., the cross-section times the velocity averaged over the velocity distribution function. Although one usually assumes a Maxwellian velocity distribution for the colliding particles, in most cases electrons, this is not required necessarily for the measurements described in the following, since one also obtains this distribution function for the electrons directly from the spectrum of laser light scattered by the plasma.

The following discussions will be restricted to the techniques outlined above. For reviews of the electron beam methods, the reader is referred to Kieffer and Dunn (1966), Harrison (1968), Heddle and Keesing (1968) and Moiseiwitsch and Smith (1968).

\section{Principle of the Measurements}

The principle of these measurements is rather straightforward. In general, the total emission coefficient of an optically thin spectral line arising from spontaneous transitions between bound levels $p$ and $q$ of an ion is given, per unit volume, per steradian and as a function of time $t$, by 


$$
\varepsilon(p \rightarrow q, t)=\frac{h v}{4 \pi} A(p \rightarrow q) N(p, t) \stackrel{W}{\mathrm{~cm}^{3} \mathrm{sr}},
$$

where $h v$ is the energy of the emitted photons in Joules, $A$ is the atomic transition probability and $N(p, t)$ is the population density of the upper level. It is important to keep in mind that the only quantity obtained from any measurement of the emission coefficient is this population density $N(p, t)$ of the ions in the upper level $p$ (assuming of course, that $A$ is known). Any further deduction of specific rate coefficients depends solely on the possibility of connecting $N(p, t)$ with the population densities of strongly populated states through appropriate models. High density plasmas are of no use here, because the excited state populations are governed by collisional processes between each other, which is sufficient for establishing a thermodynamic equilibrium population distribution between them. At low electron densities, on the other hand, a coronal model holds, i.e., the steady-state population of an excited level is determined by a balance between the sum of collisional transitions into that state and the sum of all radiative decay rates. For further considerations we assume that besides the ground state $(g)$ only one excited level $(m)$ is strongly populated [ $(m)$ will quite often be a metastable level]. Equation (1) can then be written:

$$
\begin{aligned}
\varepsilon(p \rightarrow q, t)=\frac{h v}{4 \pi} \frac{A(p \rightarrow q)}{\sum_{r<p} A(p \rightarrow r)} N(t)[N(g, t) X(g \rightarrow p)+ & \\
& +N(m, t) X(m \rightarrow p)]
\end{aligned}
$$

where $N(t)$ is the electron density, $N(g, t)$ and $N(m, t)$ are the population densities of the levels $(g)$ and $(m)$, and the $X$ 's are the respective collisional rate coefficients from these levels to the level $p$. The rate coefficients are, of course, implicitly also a function of time via a varying electron temperature. If we define $k$ as the ratio of impurity ions of one species relative to the electron density (obtained from the ratio of the initial filling pressures in the discharge) and $f(t)$ as the fraction of impurity atoms in the specific ionization stage, we obtain

$$
N(g, t)+N(m, t)=f(t) k N(t) .
$$

We further introduce the ratio $R(t)=N(m, t) / N(g, t)$ of the two population densities and obtain finally

$$
\varepsilon(p \rightarrow q, t)=\frac{h v}{4 \pi} \frac{A(p \rightarrow q)}{\sum_{r<p} A(p \rightarrow r)} k N^{2}(t) f(t)\left[\begin{array}{c}
X(g \rightarrow p)+R(t) X(m \rightarrow p) \\
R(t)+1
\end{array}\right]
$$

The average excitation rate coefficient in square brackets above is thus the final quantity, which can be deduced from the measured emission coefficient. Even if $R$ is known, unique results can thus be expected only in the limits $R \rightarrow 0$ and $R \rightarrow \infty$ or for those cases, where one of the two rate coefficients is negligibly small. 
Let us consider now the case $R \rightarrow 0$. The emission coefficient is then given by

$$
\varepsilon(t)=\text { const } \times N(t) X(t) N(g, t) .
$$

For a constant electron temperature and density the time history of the emission coefficient reflects thus exactly the time history of the ground state population. Otherwise, the time variation of $N$ and $X$ can readily be corrected for even if the absolute value of $X$ is not known too accurately. Its time dependence should be represented sufficiently well by the effective Gaunt-factor approximation (Van Regemorter, 1962; Seaton, 1962a), at least for the usually small temperature variations occurring during the emission of many of the lines in transient plasmas.

In this model, the concentration $N_{j}$ of the $j$ th ionization stage [in our approximation identical to the population $N_{j}(g, t)$ of the ground state] is determined by the following rate equation:

$$
\frac{\mathrm{d} N_{j}}{\mathrm{~d} t}=N\left(N_{j-1} I_{j-1}-N_{j} I_{j}+N_{j+1} \alpha_{j+1}-N_{j} \alpha_{j}\right)+\text { source terms },
$$

where $I_{j}$ is the rate coefficient for ionization and $\alpha_{j}$ that for recombination. While in a steady plasma the distribution between two successive ionization stages is determined by the famous corona relation $\left(N_{j+1} / N_{j}=I_{j} / \alpha_{j+1}\right)$, for our investigations those transient situations are of importance where a plasma is heated so rapidly that its ions are far from their ionization equilibrium. In this case recombination rates are usually so small that they can be safely neglected, and the time histories of the ions are then solely governed by the ionization rates. The principle of the measurement of ionization rate coefficients is therefore to solve the whole set of coupled rate Equations (6) using a computer program. The measured electron density and temperature as well as theoretical rate coefficients having approximately the correct temperature dependence are used as input. The theoretical ionization rate coefficients are then varied until the calculated time histories agree with the observed ones.

Equation (6) remains valid if ionization also occurs from excited levels: the products $N N_{j} I_{j}$ represent then the sum of the ionization rates from the ground state and all the populated excited states. Similarly to the excitation rates, only average ionization rate coefficients can be obtained in these cases.

The determination of excitation rates is not completely independent from the measurement of the ionization rates. According to Equation (4) the fraction $f(t)$ of the impurity atoms in the ionization stage of interest has to be known, and at present no reliable experimental method exists for its determination. It is obtained, therefore, usually also from the solutions of the coupled set of rate Equations (6). Fortunately it is found that the fraction $f(t)$ is relatively insensitive to the values adopted for the ionization coefficients at the time of the peak concentration. For the higher ionization stages one has to check, of course, whether the neglect of recombination is still possible, if not, appropriate corrections have to be made.

The step from Equation (1) to Equation (2) required the assumption of a steadystate population of the excited level $p$ with respect to the ground state $(g)$ and level $(m)$. 
This is usually justified in most practical cases, the relaxation time of the excited state population density being determined by the radiative decay rate. This time scale is much shorter than the time scale for changes in the ground state population due to ionization. However, the above assumption may not hold for metastable levels, the relaxation times being dominated here by collisional processes (Johnston III and Kunze, 1971).

Besides using the time history and the absolute intensity of spectral lines for measuring collisional rate coefficients, the spectral width of suitably chosen lines may also yield such quantities. In the impact approximation to the general theory of line broadening by charged particles the half-width of a spectral line (in frequency units) can be written as (Baranger, 1958; Griem, 1972)

$$
w=\frac{1}{2} N\left(\left\langle\sigma_{p} v\right\rangle+\left\langle\sigma_{q} v\right\rangle\right)-\text { interference term },
$$

where the cross-sections are the total cross-sections for elastic and inelastic scattering of electrons on atoms in upper $(p)$ and lower states $(q)$ of the line. The interference term is usually small (less than $10 \%$ ), and the term resulting from scattering on the lower states can also be neglected in many cases. The width of the emission line is thus essentially determined by the total cross section for scattering of electrons by ions in the upper state. For high electron temperatures the inelastic cross- sections dominate the elastic ones, the spectral widths thus giving directly the sum of the collisional rate coefficients from close-by levels.

It may seem, at first, somewhat surprising that this possibility of obtaining collisional rate coefficients has not been used extensively. However, for highly charged ions the broadening of suitable spectral lines by electron impacts not only becomes relatively small at the electron densities usually obtained in hot plasma devices, but the dominant broadening occurs through Doppler effect caused by the thermal motion of the ions.

Table I summarizes the experimental possibilities. The first two techniques require transient plasmas, whereas the last one may also be used on quiescent plasmas.

\section{TABLE I}

Emission lines Collisional rate coefficients

Time history

Collisional ionization from ground state and metastable levels

Absolute intensity

Collisional excitation from ground state and metastable levels

Spectral width Collisional transitions from close-by levels

\section{Theoretical Rate Coefficient}

The most recent review of the principles of different theoretical methods for calcula- 
ting excitation and ionization cross sections as well as a discussion of the reliability and the accuracy of the various approximations has been published by Bely and Van Regemorter (1970). A review article dealing specifically with ionization processes appeared by Rudge (1968).

\subsection{EXCITATION}

In general, if one desires a high accuracy of the cross-sections one has to rely on calculations carried out specifically for the transition of interest. However, for optically allowed transitions two methods are available, which yield the cross-sections as functions of the oscillator strength and the energy levels of the transition.

The first general formula is due to Van Regemorter (1962) and Seaton (1962a) and is based on the Bethe-Born approximation. Van Regemorter derived the effective Gaunt factor empirically by comparison with all cross section data then available. In this approximation the rate coefficient can be written

$$
X(q \rightarrow p)=1.60 \times 10^{-5} \frac{f_{p q}\langle\bar{g}\rangle}{\Delta E(k T)^{1 / 2}} e^{-\Delta E / k \mathrm{~T}} \mathrm{~cm}^{3} \mathrm{~s}^{-1},
$$

where $f_{p q}$ is the absorption oscillator strength, $\Delta E$ is the energy difference between levels $p$ and $q$, and $k T$ is the electron temperature, both in $\mathrm{eV}$; the effective Gaunt factor $\langle\bar{g}\rangle$ averaged over a Maxwellian velocity distribution is given by Van Regemorter (1962) and can also be found in Allen (1963). Although this approximation is being used widely, more recent calculations have shown that this very convenient procedure must be used with caution. It has been found, e.g., that for transitions between levels of identical principal quantum numbers the effective Gaunt factor approximation yields rate coefficients which are about a factor of 2 too small. In addtion, the Gaunt factor depends also on the ionic charge.

Better results can be obtained with an impact parameter method introduced for atom-electron collisions by Seaton (1962b) and generalized to positive ions by Burgess (1964). The complex functions are tabulated since the results cannot be expressed in a simple analytic form.

Cross-sections in the impact approximation have been calculated to some extent specifically in connection with the line-broadening theory. A general review of this subject can be found in the monograph by Griem (1972). A convenient analytical expression can be obtained for the collisional rate coefficient for transitions between levels which are nearly degenerate $(\Delta n=0)$. In the high-temperature limit this rate coefficient from level $l$ to $l-1$ is (Griem et al., 1962)

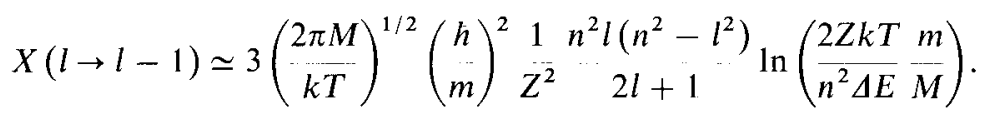

$Z$ is the effective charge on the outer electron, $m$ the electron mass and $M$ the mass of the colliding charged particle, i.e., $M=m$ for electron collisions; $n, l$, and $\hbar$ have the usual meaning. 


\subsection{IONIZATION}

Ionization cross-sections for positive ions have been calculated using quite a number of approximations although Bely and Van Regemorter (1970) conclude that the Coulomb-Born II and the Born-exchange are the best approximations presently available. In contrast to the excitation cross-section one can easily obtain a quick and reliable estimate. This is based essentially on the classical Thomson theory which suggests that the reduced ionization cross sections should be about the same for all species. This is substantiated by Bely and Faucher (1972) who present a universal function for ionization of atoms by structureless charged particles of arbitrary mass and charge.

Quite a number of empirical formulae for ionization have been proposed [Elwert, 1952; Drawin, 1961; Wilson and White 1964; Kolb and Lupton 1964, (the latter two as given by McWhirter, 1965); Burgess and Seaton, 1964; Lotz, 1967a; Kunze, 1971a]. Lotz $(1967 b, 1968)$ took into account all experimental data on cross-sections and presents his results as an extensive and convenient tabular compilation.

\section{Experimental Methods}

\subsection{THETA-PINCH}

If one looks at the measurements of collisional rate coefficients done so far using laboratory plasmas, most of them used plasmas produced in a so-called theta-pinch device, although a wide range of plasma sources is now available for spectroscopic studies, as was reviewed recently by Gabriel (1970). Most theta-pinch devices have been developed during the last decade in connection with controlled fusion experiments. A recent description of these machines is given, for example, by Niblett (1970). A theta-pinch designed specifically for this type of spectroscopic studies was built by DeSilva and Kunze (1968).

A theta-pinch consists essentially of a discharge tube having wrapped around it a single turn coil (see Figure 1). The discharge tube is filled usually with hydrogen plus a small amount of the desired atoms. After the gas has been pre-ionized, a low-inductance capacitor bank is discharged through this coil. The azimuthal current induces an

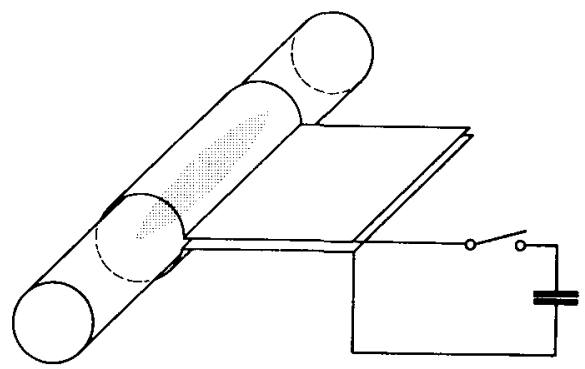

Fig. 1. Schematic theta-pinch geometry. 
azimuthal current in the discharge tube which compresses the plasma, and through a combination of shock, Joule and adiabatic heating a usually cigar-shaped hot plasma is produced in the middle of the coil. The electron temperature reaches a few hundred $\mathrm{eV}$ and is limited by cooling through axial thermal conduction (Green et al., 1967). Ion temperatures are usually higher (in the $\mathrm{keV}$ range) and increase also with increasing charge of the impurity ions. The densities obtained range typically from about $10^{15}$ to $10^{17} \mathrm{~cm}^{-3}$. The lifetime of the hot plasma is limited in many cases by the period of the discharge current if the coil is made sufficiently long to reduce plasma end-losses. This technical limit can be somewhat increased by short-circuiting the capacitor bank at current maximum (usually referred to as crowbarring) which yields an exponentially decaying current through the coil. The lifetimes of the plasmas are of the order of microseconds for smaller devices, but can vary between 10 and $100 \mu \mathrm{sec}$ for the large devices used in nuclear fusion studies.

\subsection{Determination OF Plasma PaRAmeters}

For the determination of plasma parameters spectroscopic methods are not recommendable in connection with these measurements. A convenient non-spectroscopic technique free of most ambiguities has been perfected over the last decade and is now applied routinely to the diagnostics of laboratory plasmas; i.e. the spectral measurement of laser radiaton scattered by the free electrons in the plasma. A description of this technique can be found in review articles by Kunze (1968) and by DeSilva and Goldenbaum (1970). In the simplest case when the electrons scatter completely independently of each other (the classical Thomson scattering case) the Doppler broadened spectrum of the scattered light reflects exactly the velocity distribution function of the electrons in the direction of the scattering vector. One obtains thus not

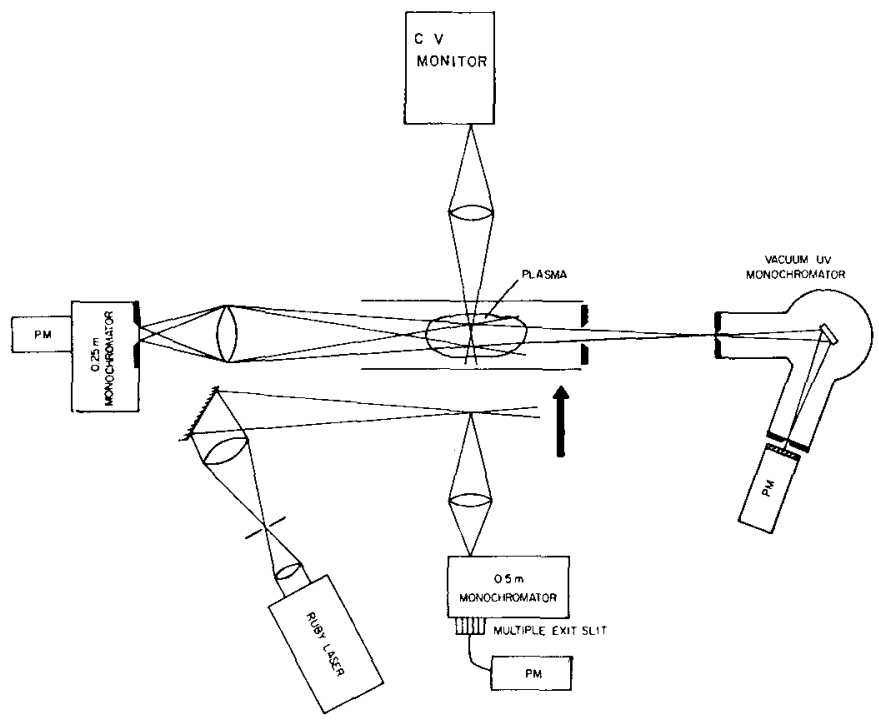

Fig. 2. Schematic showing typical experimental setup. 
only the electron temperature but one also has a check that indeed a Maxwellian velocity distribution exists. This scattering regime can be reached by proper choice of the scattering angle, and in hot plasmas a scattering angle of $90^{\circ}$ is usually sufficient. The total intensity of the scattered light yields the electron density.

A typical experimental setup can be seen in Figure 2. The beam from a high-power Q-switched ruby laser is directed along the axis of the discharge tube and focused in the midplane of the coil, which is slotted for observation. Radiation scattered by the plas$\mathrm{ma}$ is observed at $90^{\circ}$ and passed through a multichannel spectrometer, which allows the simultaneous measurement of several portions of the spectrum. The laser head and the detecting system are mounted on a common carriage, permitting easy scanning of the scattering volume along a diameter of the plasma column. This arrangement has the advantage that rather local plasma parameters can be deduced, and Figure 3 shows as an example results thus obtained (Kunze, 1971a).

The ion temperatures of the impurity ions are deduced from the Doppler widths of suitable spectral lines. They are needed to estimate optical depth corrections on the absolute intensities of measured spectral lines. In this respect it is advantageous that the ion temperatures are usually much higher than the electron temperatures in theta-pinch devices. Finally, spectroscopic observations yield results integrated along the line of sight. If one observes the line emission through the ends of the discharge tube, the length of the plasma column in axial direction has to be known. It can be obtained by observing suitable line radiation through holes in the coil.
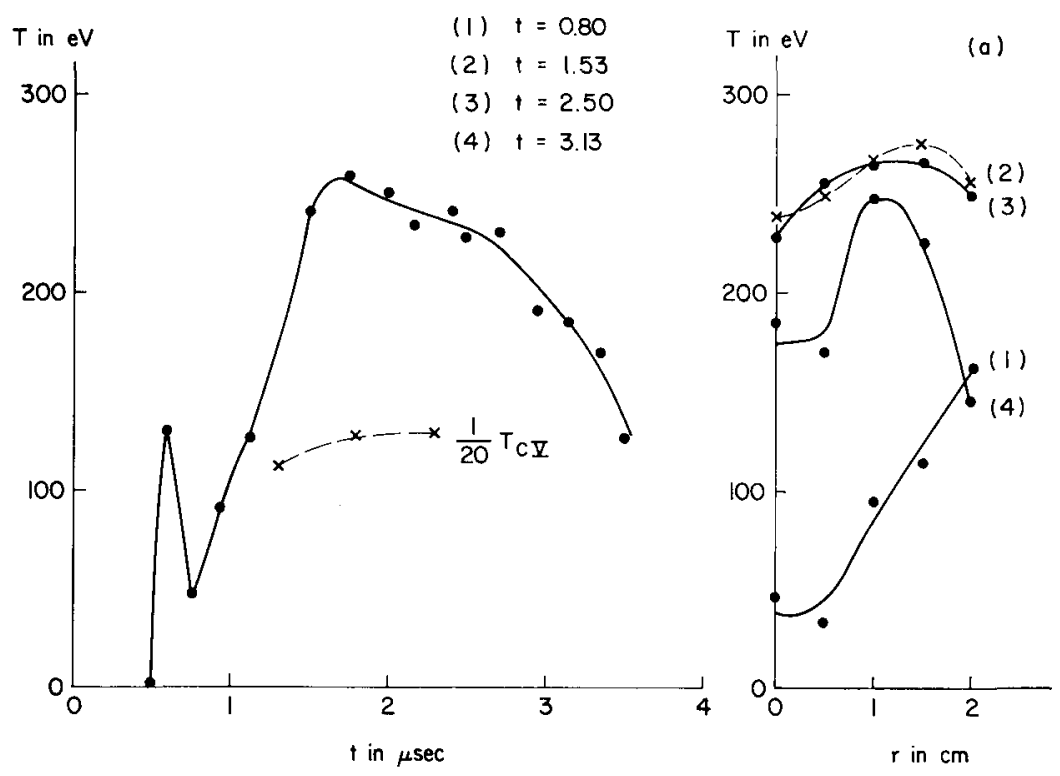

Figs. 3a-b. Local plasma parameters in the midplane of a theta-pinch coil, obtained as a function of time $t$ and radius $r$ from Thomson-scattering measurements: (a) for electron temperature $T$, and (b) for electron density $N$ (from Kunze, 1971a). 

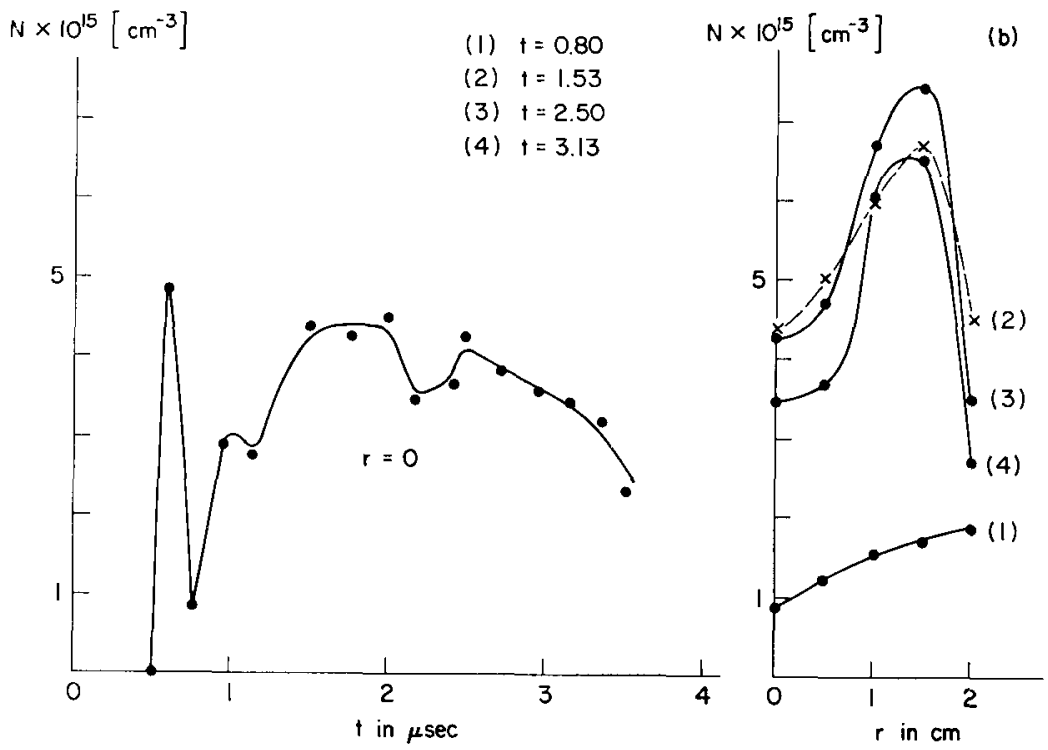

Fig. $3 b$.

\subsection{SPECTROSCOPIC MEASUREMENTS}

Strong lines from highly ionized atoms are usually in the vacuum-ultraviolet and X-ray wavelength regions. One crucial point in these spectroscopic measurements is the absolute sensitivity calibration of the proper spectroscopic instruments, i.e., vacuumultraviolet normal-incidence-, grazing-incidence- and Bragg-crystal-monochromators. For the short wavelength region techniques used in X-ray spectroscopy and extended by Morgan et al. (1968) up to about $100 \AA$ can be used. For the longer wavelengths, no generally accepted radiation standard is available, although some have been proposed (e.g., Newburgh et al., 1962; Bogen et al., 1968). Radiation from a synchrotron provides an excellent standard (Hinteregger, 1965; Thimm, 1971), however, the experimental procedures are not simple and a synchrotron is also not everywhere at hand.

The calibration is done therefore, in most cases, using the 'branching-ratio technique', (Griffin and McWhirter, 1962; Hinnov and Hofmann, 1962). This technique is based on the observation of 2 spectral lines in the vacuum uv and the visible region both of which originate from the same upper level and neither of which is influenced by selfabsorption. The intensity ratio (in photon units) of the two lines is then simply given by the ratio of the respective transition probabilities. If one calibrates the visible line absolutely, the absolute intensity of the vacuum UV line is thus derived easily. The method can be extended to lines which originate from different fine-structure sublevels if one can prove reliably that the sublevels are populated according to their statistical weights. A table of suitable line pairs as well as a discussion of problems encountered is found in Kunze and Johnston III (197lb). These authors observed 
simultaneously both lines emitted from suitable plasmas produced in the theta-pinch through the ends of the discharge tube viewing a defined portion of the plasma (see Figure 2). The calibration could thus be done in situ and no geometrical corrections had to be applied. The branching ratio technique has now also been used for calibration at $26 \AA$ (Irons and Peacock, 1972) using laser produced plasmas, although difficulties arise from the strong dependence of intensity with distance from target. Engelhardt (1971) has recently used line ratios in the Lyman and Balmer series of hydrogen-like ions originating from different levels assuming coronal population and theoretical exitation rate coefficients. In general, however, this method has to be used with caution and will certainly not yet be accurate enough for ions other than hydrogen-like ones.

The vacuum UV monochromators are usually equipped with a scintillator-photomultiplier combination as fast detector. Because of their short decay times p-terphenyl and sodium-salicylate as well as plastic scintillators like Pilot-B or NE 102 have been used.

These experiments require a large number of individual measurements and a good monitor for the overall performance of the discharge is important. As such, the $\mathrm{CV}$ triplet at $2275 \AA$ has been found quite useful.

\section{Discussion of Experimental Results}

\subsection{IONIZATION}

Table II gives a summary of the experiments done so far on ionization rate coefficients using laboratory plasmas. The first measurements were made by Hinnov $(1966,1967)$ in a Stellarator discharge for the ions NeII to NeVII. The results for all ions are self-

TABLE II

Experiments on ionization rate coefficients

\begin{tabular}{lll}
\hline Reference & Ions & Plasma device \\
Hinnov $(1966,1967)$ & NeII-NeviII & C Stellarator \\
Kunze et al. $(1968)$ & CV & $\theta$-pinch \\
Kunze (1971a) & CIV, Nv, Ovi: Ov, NeviI & $\theta$-pinch
\end{tabular}

consistent; however, if we compare the rate coefficients with the tabulations by Lotz (1967a), we find the experimental values are a factor of 1.5 to 3 below the tabulated ones, in all cases beyond the estimated experimental accuracy. The largest deviations occur for the lowest and the highest ionization stages.

The second experiment was done on $\mathrm{CV}$ as an example of helium-like ions (Kunze et al., 1968). Agreement to better than $25 \%$ was obtained between experiment and tabulations by Lotz as well as other theoretical calculations (Beigman and Vainshtein, 1967) and semi-empirical formulae (e.g., see Kunze, 1971a).

The third experiment was done to study specifically ions of the lithium and beryllium 
isoelectronic sequences. Complications arise for the beryllium-like ions, since they have a low-lying metastable level, which is always strongly populated; thus ionization occurs strongly also from this level, and only average rate coefficients can be obtained. At higher electron densities the situation becomes similar for lithium-like ions as the similarly low-lying 2P levels become increasingly populated. However, for the temperatures of the experiment the ionization rate coefficients from the ground state and the metastable levels, the $2 S$ and $2 P$ levels respectively, were practically the same. The general result of this experiment was that the ionization rate coefficients for lithium- and beryllium-like ions were on the average only $60 \%$ of the values as quoted by Lotz, or as were obtained from a semi-empirical formula which was based on the theoretical calculations by Rudge and Schwartz (1966) for hydrogen-like ions. This deviation was within the estimated experimental accuracy and it was noted that possible systematic errors, like ions in the low-density plasma outside the hot core, would tend to yield too low values.

Nevertheless, if we consider this experiment together with Hinnov's $(1966,1967)$ measurements, both could indicate that for ions having more than two electrons the theoretical as well as the semi-empirical results might give values about a factor of 1.5 to 2 too large. Further experiments should clarify the situation. The theory is apparently sufficiently correct for helium-like ions.

\subsection{EXCITATION}

Table III finally summarizes the experiments, in chronological order, in which rate coefficients for excitation have been obtained. Hinnov (1966) measured the absolute intensities of various resonance multiplets from NeII to NeVIII in a stellarator discharge and deduced the corresponding rate coefficients. He compared his experimental results with theoretical coefficients based on the Bethe-Born approximation which differed from Seaton's and Van Regemorter's effective Gaunt factor formula [Equation (8)] inasmuch as Hinnov's 'Gaunt factor' showed a somewhat different dependence on the energy. This difference, however, never exceeded $30 \%$ from threshold to

TABLE III

Experiments on excitation rate coefficients

\begin{tabular}{|c|c|c|}
\hline Reference & Ions & Plasma Device \\
\hline Hinnov (1966) & $\mathrm{Ne}$ II-Ne viII & C Stellarator \\
\hline Johnson (1967) & $\mathrm{He}$ & C Stellarator \\
\hline Elton and Köppendörfer (1967) & OVII & $\theta$-pinch \\
\hline Kunze et al. (1968) & $\mathrm{CV}$ & $\theta$-pinch \\
\hline Johnson and Hinnov (1969) & $\mathrm{He}$ & C Stellarator \\
\hline Boland et al. (1970) & Nv & ZETA \\
\hline Kunze and Johnston III (1971) & Nv, O vi, Ne vilI & $\theta$-pinch \\
\hline Tondello and McWhirter (1971) & $\mathrm{NeVII}$ & $\theta$-pinch \\
\hline Kunze (1971b) & NeviII & $\theta$-pinch \\
\hline Johnston III and Kunze (1971) & Niv, Oy, NeviI, SixI & $\theta$-pinch \\
\hline Engelhardt et al. (1972) & Cv, Nvi, OvII, FviII, NeIx & $\theta$-pinch \\
\hline Gabriel et al. (1972) & CV, NVI, OVII & 0 -pinch \\
\hline
\end{tabular}


about 100 times threshold. The experimental results agree well with the theoretical predictions (except for $\mathrm{Ne}$ II), although one has to keep in mind that the oscillator strengths used have an estimated uncertainty of larger than 50\% (Wiese et al., 1966).

Johnson's (1967) measurements on neutral helium actually do not belong in this discussion of rate coefficients of highly charged ions. However, the experiment was performed using the same techniques and a plasma produced in a stellarator discharge, so it is listed for completeness. This comment is true also for the second experiment on helium by Johnson and Hinnov (1969).

The other experiments can be grouped according to the ions studied and will be discussed therefore correspondingly.

\subsubsection{Lithium-like Ions}

Lithium-like ions have been studied by two groups; at the Culham Laboratory (Boland et al., 1970) and at the University of Maryland (Kunze and Johnston III, 1971; Kunze, 1971b). They have the tremendous advantage that they have a simple electronic structure and are thus rather amenable to theoretical calculations. Quite extensive calculations have been done especially by Bely and Petrini for several ions of the sequence using the Coulomb-Born approximation (Bely, 1966a; Bely, 1966b; Bely and Petrini, 1970). Burke et al. (1966) have used several approximations including two variants of the close coupling method for Nv. Very recently Flower (1971) calculated the cross-sections for NV and SixII in the distorted wave approximation and gives also a comparison of his results with those obtained using the other approximations. In general, there is good overall agreement.

The excitation rate coefficients for 5 transitions in $\mathrm{Nv}$ have been measured by the Culham group at one temperature using the plasma device ZETA. After the measured excitation rate coefficient for the $2 s \rightarrow 2 p$ transition has been normalized to the theoretical value, the measured rates for the other transitions agree with the calculations of Burke et al. (1966) to within $20 \%$. The Maryland group using a theta-pinch device measured the rate coefficients for all transitions from the ground state to the $n=2,3$ and 4 levels in $\mathrm{Nv}, \mathrm{OVI}$ and $\mathrm{Ne}$ vil at three different discharge conditions. Due to the number of individual measurements necessary to obtain one rate coefficient, the maximum error of any individual rate coefficient was estimated to be a factor of 2; however, if one compares all experimental values with the theoretical ones as given for example by Bely $(1966 \mathrm{a}, \mathrm{b})$ one can deduce for each transition in all three ions a standard deviation of the experimental values from the respective theoretical ones. This standard deviation is found to be less than $30 \%$ for the excitation rate coefficients to the $n=2$ and $n=3$ levels, and less than $40 \%$ for excitation to the $4 s$ levels. The rates to the $4 p$ and $4 d$ levels are on the average only about $60 \%$ of the theoretical ones. An interesting result was obtained for the excitation to the $4 f$ levels in the Nevill. This excitation transition corresponds to a $\Delta l=3$ change of the angular momentum of the ion, and the magnitude of this rate was found to be equal to that for the corresponding $\Delta l=1$ dipole rate to the $4 p$ level. The electron temperature in this experiment was of the order of the excitation energy. 
Some of the measurements by Kunze and Johnston III (1971) were made at higher electron densities which lead to a stronger population of the $2 p$ levels. In these cases a significant population of the $n p$ and $n d$ levels occurred via the $2 p$ levels, the population of the $n s$ levels from the $2 p$ levels, however, being always sufficiently small compared to that from the ground state. According to Equation (4), only averaged excitation rate coefficients could be deduced in these cases.

The two experiments thus confirm the various theoretical results, that the effective Gaunt factor approximation yields rate coefficients about a factor of two too small for the $2 s \rightarrow 2 p$ transition $(\Delta n=0)$ at the electron temperatures at which these ions usually exist in laboratory or astrophysical plasmas. The predictions of the Gaunt factor approximation for transition to the $3 p$ and $4 p$ levels $(\Delta n \neq 0)$ agree to better than $30 \%$ with the other theoretical values and the experiments.

\subsubsection{Helium-like Ions}

If we go to ions having two outer electrons, we come to the ions belonging to the helium and beryllium isoelectronic sequences, which are characterized by having metastable levels.

Specific calculations have been carried out so far only by Bely (1968) for OvII; however, some cros-sections for various intercombination transitions in helium-like ions have been estimated by Burgess et al. (1970) from their calculations of hydrogenlike ions. A comparison of the available data as well as a discussion of the important processes in helium-like ions at low astrophysical and high laboratory densities can be found in Gabriel and Jordan (1972).

First experiments on helium-like ions were carried out by Elton and Köppendörfer (1967) on Ovil and by Kunze et al. (1968) on CV. After development of a modified coronal model for helium-like ions the absolute rate coefficients for excitation of the $n=2$ singlet levels were derived for $\mathrm{CV}$ and $\mathrm{O}$ VII to an experimental accuracy of $40 \%$ and a factor of 2 , respectively. The results agreed to within the experimental errors with rates calculated according to Equation (8). Kunze, Gabriel and Griem measured also the intensity ratio of resonance line and intercombination line as a function of the electron density and attempted to deduce from their results and using the model mentioned above the triplet to singlet collisional exchange rate and the ratio of the excitation rates from the ground state to the $n=2$ singlet and triplet levels. In a series of recent collaborative experiments between the Astrophysical Research Unit in Culham and the University of Maryland (Gabriel et al., 1972) the density range has not only been extended but the measurements have been carried out now also for other ions. Utilizing better theoretical data now available an improved coronal model for helium-like ions in laboratory plasmas is being used for the interpretation of these measurements.

Engelhardt et al. (1972) measured the intensity ratios of the three $2^{3} P \rightarrow 2^{3} S$ transitions for various helium-like ions as a function of the electron density and obtained the collisional rate coefficients between the triplet $P$ and $S$ states. 


\subsubsection{Beryllium-like Ions}

Two very recent experiments by Tondello and McWhirter (1971) and Johnston III and Kunze (1971) deal with beryllium-like ions.

Specific calculations of excitation cross-sections for these ions have been computed so far only by Osterbrock (1970), who solved the coupled integro-differential equations for the radial waves of the free electrons, by Eissner (1971) with the distorted wave method and by Nussbaumer (1971)using the impact parameter method (with coefficients from the tables of Burgess, 1964).

The metastable level in beryllium-like ions is low-lying, as a consequence of which this level is always strongly populated at laboratory as well as at many astrophysical plasma densities. Excitation of the higher levels occurs thus from both the ground state and the metastable level, and for the deduction of any rate coefficient it is necessary to obtain the relative population density distribution $R$ between the two. At present no very reliable theoretical possibility exists to obtain this ratio $R$ for many laboratory plasmas. The situation would become simple only for high-density steadystate plasmas where a Boltzmann distribution between the two states would prevail. In many transient laboratory plasmas, however, no steady-state population for the metastable levels is reached. The situation is further complicated by the fact that the metastable level is also populated directly by ionization of the preceding boron-like ionization stage. (About one-third of the ionizing collisions from the ground state of the boron-like ion should lead to the metastable triplet level of the beryllium-like ion; however, all ionizing collisions from the metastable quartet state of the boronlike ion will lead finally to the metastable triplet level.)

At present, it appears therefore best to rely on the experiment. Two possibilities exist for obtaining this ratio $R$. Figure 4 shows a partial term diagram of beryllium-like ions containing all $n=2$ levels. One method is to measure absolutely
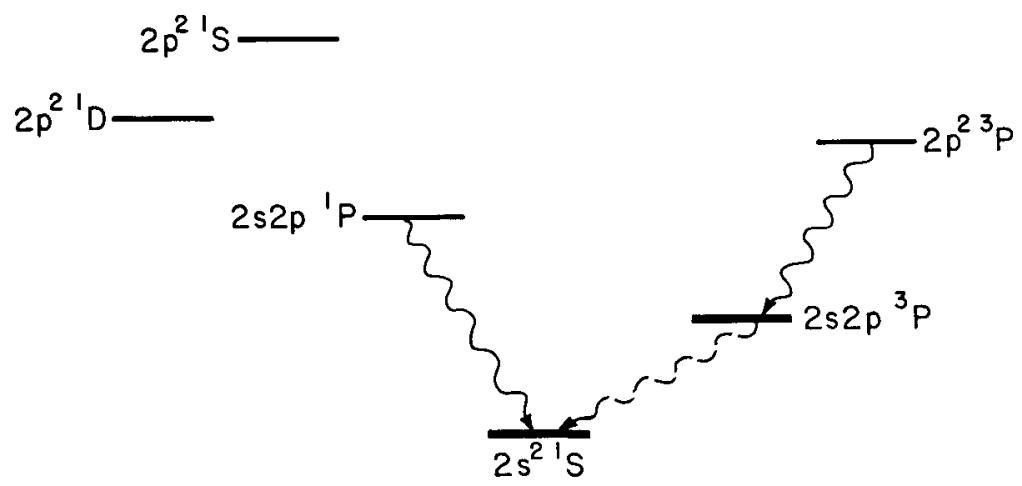

Fig. 4. Partial term diagram for beryllium-like ions. 
the emission coefficient of the intercombination transition $2 s 2 p{ }^{3} P_{1} \rightarrow 2 s^{2}{ }^{1} S_{0}$, which, unfortunately, is extremely weak in laboratory plasmas, if observable at all. From this measurement the Maryland group obtained $R=0.92$ for Ne vil using the oscillator strength for the intercombination line as given by Garstang and Shamey (1967). However, more recent calcalutions by Nussbaumer (1971) including configuration interaction give transition probabilities substantially lower. Using his value for NevII the value of $R$ would have to be $R \simeq 6.1$ for the case mentioned above. (This $R$-value is now extremely sensitive to the measured intensity of the intercombination transition. With a compounded error of $35 \%$ on the emission coefficient $R$ could thus be as low as $R \simeq 1.4$, whereas in the other direction it could of course not exceed the theoretical Boltzmann limit $R \simeq 8$.6.)

The second experimental possibility is based on the following considerations: the upper level of the resonance line will be populated mostly by collisions from the ground state and the upper level of the $2 p^{2}{ }^{3} P \rightarrow 2 s 2 p^{3} P$ transition by collisions from the metastable level. The intensity ratio of the two lines (in photon units) is thus simply given by

$$
\frac{I\left(2 s 2 p^{1} P \rightarrow 2 s^{2}{ }^{1} S\right)}{I\left(2 p^{2}{ }^{3} P \rightarrow 2 s 2 p^{3} P\right)} \simeq \frac{1 X\left(2 s^{2}{ }^{1} S \rightarrow 2 s 2 p^{1} P\right)}{R X\left(2 s 2 p^{3} P \rightarrow 2 p^{2}{ }^{3} P\right)}
$$

i.e., if the two excitation rates were known, the ratio $R$ could be derived directly from the intensity ratio of the two lines. This method has the advantage, that it does not require a difficult absolute intensity measurement in the vacuum-ultraviolet wavelength region. Relative measurements can be done with much higher accuracy. In addition, one also needs only the ratio of the two excitation rate coefficients. (Corrections for cascading and exchange collisions can be applied, of course.)

For the case discussed above, the effective Gaunt factor approximation predicts a ratio of 2 for the excitation rates as do specific calculations by Eissner (1971). If one uses this value, $R=1.75$ is obtained. In view of the larger uncertainties still associated with the first method, it is suggested that this method yields the more reliable results. This value is lower than the value of $R \approx 8$ derived by the Culham group from theoretical considerations.

In principle, considered from a different point of view, a combination of both these methods constitutes nothing else but a measurement of the transition probability of the intercombination transition. For the case above one would obtain $A \simeq 2.2 \times 10^{4} \mathrm{~s}^{-1}$ with a probable error of about $50 \%$

With the ratio $R$ known, excitation rates to the higher level can be derived. In the evaluation of rate coefficients one can make the additional assumption (supported by theoretical results of Eissner, 1971) that collisional transitions involving spin change are weaker or negligible compared to equivalent ones with no spin change, i.e., $X(g \rightarrow p)$ or $X(m \rightarrow p)$ in Equation (4) were neglected where appropriate.

In view of the discussion above, the final rate coefficients as quoted by Johnston III and Kunze (1971) should now indeed be multiplied by a factor of 1.4 for the singlet 
system and divided by a factor of 1.3 for the triplet system as was already indicated in the discussion section of that paper.

Of interest is a comparison of the experimental results with coefficients obtained in the effective Gaunt factor approximation. Let us consider, for example, the $2 s \rightarrow 3 p$ transitions. Agreement to within the experimental error of 2 is obtained only if one uses oscillator strengths which have been calculated taking into account configuration mixing (Nussbaumer, 1969, 1971; Friedrich and Trefftz, 1969). This suggests that the effective Gaunt factor approximation gives rate coefficients for $\Delta n \neq 0$ transitions to better than a factor of two for beryllium-like ions also if the correct oscillator strengths are known.

\section{Conclusion}

Various experiments have obtained collisional rate coefficients for excitation and ionization of highly ionized atoms from the analysis of line radiation emitted by these ions in hot well-diagnosed laboratory plasmas. Although quite a number of individual measurements had to be done before an individual rate coefficient could be deduced, no principal difficulties were encountered with these methods. In a recent experiment at the University of Maryland, however, it was attempted to apply these techniques also to heavy ions like argon. Surprisingly it was found that the heavy argon atoms do not mix evenly with the hot plasma. Although this effect might very well be connected with the specific low-magnetic field theta-pinch device used, the observation suggests caution in similar experiments.

\section{Acknowledgement}

This work was supported by the Atomic Energy Commission and the Office of Naval Research.

\section{References}

Allen, C. W.: 1963, Astrophysical Quantities, 2nd ed. Athlone University of London, London. Baranger, M.: 1958, Phys. Rev. 112, 855.

Beigman, I. L. and Vainshtein, L. A.: 1967, Astron. Zh. 44, 889. [1968, Soviet Astron. AJ 11, 712].

Bely, O.: 1966a, Proc. Phys. Soc. (London) 88, 587; Ann. Astrophys. 29, 121.

Bely, O.: 1966b, Ann. Astrophys. 29, 683.

Bely, O.: 1968, Phys. Letters 26A, 408.

Bely, O. and Petrini, D.: 1970, Astron. Astrophys. 6, 318.

Bely, O. and Van Regemorter, H.: 1970, Ann. Rev. Astron. Astrophys. 8, 329.

Bely, O. and Faucher, P.: 1972, this issue, p. 588.

Bogen, P., Rusbüldt, D., and Schlüter, J.: 1968, Symposium on Calibration Methods in the Ultraviolet and X-Ray Regions of the Spectrum, Munich, Report ESRO-SP33, p. 53.

Boland, B., Jahoda, F., Jones, T. J. L., and McWhirter, R. W. P.: 1970, J. Phys. B: Atom. Molec. Phys. 3, 1134.

Burgess, A.: 1964, Proc. Symp. on Atomic Collision Processes in Plasmas, U.K. Atomic Energy Authority Report AERE-R4818, p. 63.

Burgess, A. and Seaton, M. J.: 1964, Monthly Notices Roy Astron. Soc. 127, 335.

Burgess, A., Hummer, D. G., and Tully, J. A.: 1970, Phil. Trans. Roy. Soc. (London) 79, 1105.

Burke, P. G., Tait, J. H., and Lewis, B. A.: 1966, Proc. Phys. Soc. (London) 87, 209.

DeSilva, A. W. and Kunze, H.-J.: 1968, J. Appl. Phys. 39, 2458. 
DeSilva, A. W. and Goldenbaum, G. C.: 1970, in L. Marton(ed.), Methods of Experimental Physics, Vol. 9A, Academic Press, New York.

Drawin, H. W.: 1961, Z. Physik 164, 513 with corrigenda in Z. Physik 168 (1962), 238.

Eissner, W.: 1971, to be published.

Elton, R. C.: 1970, in L. Marton(ed.), Methods of Experimental Physics, Vol. 9A, Academic Press, New York.

Elton, R. C. and Köppendörfer, W. W.: 1967, Phys. Rev. 160, 194.

Elwert, G.: 1952, Z. Naturforsch. 7a, 432.

Engelhardt, W.: 1971, Z. Physik 244, 70.

Engelhardt, W., Köppendörfer, W. W., and Sommer, J.: 1972, to be published.

Flower, D. R.: 1971, J. Phys. B: Atom. Molec. Phys. 4, 697.

Friedrich, H. and Trefftz, E.: 1969, Report MPI-PAE/Astro 29, Max-Planck-Inst. Physik und Astrophysik, Munich.

Gabriel, A. H.: 1970, Nucl. Instr. Methods 90, 157.

Gabriel, A. H. and Jordan, C.: 1972, in E. W. McDaniel and M. R. C. McDowell (eds.), Case Studies in Atomic Collision Physics, Vol. II, North-Holland Publ. Co., Amsterdam.

Gabriel, A. H., Paget, T. M., and Kunze, H.-J.: 1972, to be published.

Garstang, R. H. and Shamey, L. J.: 1967, Astrophys. J. 148, 665.

Green, T. S., Fisher, D. L., Gabriel, A. H., Morgan, F. J., and Newton, A. A.: 1967, Phys. Fluids $10,1663$.

Griem, H. R.: 1964, Plasma Spectroscopy, McGraw-Hill, New York.

Griem, H. R.: 1972, Broadening of Spectral Lines by Charged Particles, Academic Press, New York, in press.

Griem, H. R., Baranger, M., Kolb, A. C., and Oertel, G. K.: 1962, Phys. Rev. 125, 177.

Griffin, W. C. and McWhirter, R. W. P.: 1962, in Proceedings of the Conference on Optical Instruments and Techniques, Chapman and Hall, London, p. 14.

Harrison, M. F. A.: 1968, in L. Marton(ed.), Methods of Experimental Physics, Vol. 7a, Academic Press, New York, p. 95.

Heddle, D. W. O. and Keesing, R. G. W.: 1968, in D. R. Bates and I. Estermann(eds.), Advances in Atomic and Molecular Physics, Vol. 4, Academic Press, New York, p. 267.

Hinnov, E.: 1966, J. Opt. Soc. Am. 56, 1179.

Hinnov, E.: 1967, J. Opt. Soc. Am. 57, 1392.

Hinnov, E. and Hofmann, F. W.: 1962, J. Opt. Soc. Am. 53, 1259.

Hinteregger, H. E.: 1965, Space Sci. Rev. 4, 461.

lrons, F. E. and Peacock, N. J.: 1972, this issue, p. 561.

Johnson, L. C.: 1967, Phys. Rev. 155, 64.

Johnson, L. C. and Hinnov, E. : 1969, Phys. Rev. 187, 143.

Johnston III, W. D. and Kunze, H.-J.: 1971, Phys. Rev. A4, 962.

Kieffer, L. J. and Dunn, G. H.: 1966, Phys, Rev. A38, 1.

Kunze, H.-J.: 1968, in W. Lochte-Holtgreven(ed.), Plasma Diagnotics, North-Holland, Publ. Co., Amsterdam, p. 550.

Kunze, H.-J.: 1971a, Phys. Rev. A3, 937.

Kunze, H.-J.: 1971b, Phys. Rev. A4, 111.

Kunze, H.-J. and Johnston III, W. D.: 1971, Phys. Rev. A3, 1384.

Kunze, H.-J., Gabriel, A. H., and Griem, H. R.: 1968, Phys. Rev. 165, 267.

Lotz, W.: 1967a, Astrophys. J. Suppl. 14, 207.

Lotz, W.: 1967b, Report IPP 1/62, Institut für Plasmaphysik, Garching bei München.

Lotz, W.: 1968, Report IPP 1/76, Institut für Plasmaphysik, Garching bei München.

McWhirter, R. W. P.: 1965, in R. H. Huddlestone and S. L. Leondard(eds.), Plasma Diagnostic Techniques, Academic Press, New York, p. 201.

Moiseiwitsch, B. L. and Smith, S. J.: 1968, Rev. Mod. Phys. 40, 238.

Morgan, F. J., Gabriel, A. H., and Burton, M. J.: 1968, J. Sci. Instrum. 1, 998.

Newburgh, R. G., Heroux, L., and Hinteregger, A. E.: 1962, Appl. Opt. 1, 733

Niblett, G. B. F.: 1970, in B. J. Rye and J. C. Taylor (eds.), Physics of Hot Plasmas, Plenum Press, New York, p. 363.

Nussbaumer, H.: 1969, Monthly Notices Roy. Astron. Soc. 145, 141.

Nussbaumer, H.: 1971, to be published. 
Osterbrock, D. E.: 1970, J. Phys. B: Atom. Molec. Phys. 3, 149.

Rudge, M. R. H.: 1968, Rev. Mod. Phys. 40, 564.

Rudge, M. R. H. and Schwartz, S. B.: 1966, Proc. Phys. Soc. (London) 88, 563.

Seaton, M. J.: 1962a, in D. R. Bates (ed.), Atomic and Molecular Processes, Academic Press, New York, p. 374.

Seaton, M. J.: 1962b, Proc. Phys. Soc. 79, 1105.

Thimm, K.: 1971, Institut für Plasmaphysik, KFA Jülich, Germany, Report Jül-748-PP.

Tondello, G. and McWhirter, R. W. P.: 1971, J. Phys. B: Atom. Molec. Phys. 4, 715.

Van Regmorter, H.: 1962, Astrophys. J. 136, 906.

Wiese, W. L., Smith, M. W., and Glennon, B. M.: 1966, Atomic Transition Probabilities, National

Bureau of Standards Publication, Washington, D. C., NSRDS-NBS4.

\section{DISCUSSION}

R.W.P. McWhirter: My comment concerns the difference between the values taken by the Culham and Maryland groups for the ratio $n(m) / n(g)$ for the Be-like ions, viz Culham $\sim 8$, Maryland $\sim 1$. Unfortunately we were at Culham unable to observe the intercombination line because it is so weak and had to calculate the ratio theoretically. With the data available at the time we found that to a factor 3 the ratio could be represented by the Boltzmann equation. More recent theoretical data suggest that ratio should be about 2 . We agree that the measurement of the intensity of the intercombination line is the better way of determining the value of $n(m)$.

A. Dalgarno: What assumptions do you make about the relative populations of the different ${ }^{3} P_{j}$ sub-levels?

H.-J. Kunze: We assume that they are populated according to their statistical weights, which will indeed be the case at the electron densities usually existing in theta-pinch plasmas.

C. Jordan: I wish to draw attention to Eissner's calculations of the collision strength for the $2 s 2 p$ ${ }^{3} P \rightarrow 2 s 2 p^{1} P$ exchange transition. He finds a value for the collision strength for this transition which is about twice that from $2 s 2 p{ }^{3} P$ to the ground state $2 s^{2}{ }^{1} S_{0}$. You have said that you neglected this process, whereas perhaps you should include it.

$H .-J$. Kunze: I was considering only the intensity ratio of the $2 s 2 p^{1} P \rightarrow 2 s^{2}{ }^{1} S$ and $2 p^{23} P \rightarrow 2 s 2 p^{3} P$ transitions, and despite the $2 s 2 p^{3} P \rightarrow 2 s 2 p^{1} P$ collision strength of Eissner's being twice as strong as that going to the ground state, the population of the $2 s 2 p^{1} P$ will be occurring predominantly from the ground state. The contribution by exchange transitions from the metastable level will be a few percent only.

D. R. Flower: I should like to point out that there remains a discrepancy of almost a factor 2 between close-coupling and Coulomb-Born calculations of the near threshold values of the $2 s-3 p$ crosssection in $\mathrm{Nv}$. Although the close-coupling method is potentially the most accurate available, the Coulomb-Born result is confirmed by some recent distorted wave calculations of my own. Furthermore, it is found that the discrepancy between the close-coupling and Coulomb-Born/distorted wave results arises for quite large values of the angular momentum of the incident electron, - a physically unexpected result. A further close-coupling calculation of this cross-section is clearly required.

H.J. Kunze: The quoted agreement between theory and experiment ( $30 \%$ standard deviation) referred to a comparison of the experiment with Coulomb-Born calculations made by Bely.

G. N. Haddad: I would like to add to Dr McWhirter's remark that I am involved with a measurement of excitation coefficients in Ne viII in a situation such as he described. For conditions in the theta-pinch at Culham the dominant process for population of the $3 \mathrm{~d}$ level is excitation from $2 p$ rather than from $2 s$. Hence a measurement of the intensity ratio of the $2 p-3 d$ line and $2 s-2 p$ line gives a measurement of the $2 p-3 d$ excitation rate which is virtually independent of other processes. Preliminary measurements of this rate coefficient are in excellent agreement with the theoretical calculations of Burke et al. and it is hoped to reduce the errors involved so that the crosssection is accurate to $+20 \%$.

H.-J. Kunze: I agree that this is possible if you have sufficiently high electron densities that the $2^{2} P_{3 / 2,1} / 2$ levels are strongly populated. Your method is very nice, since in the ratio of the two lines (as follows from Equation (2) or Equation(10)) some of the experimental uncertainties cancel. Your measurements are possible because the rate from the $2^{2} P$ levels will be about 3 times as strong as that from the ground state. In our measurements we reached $R \simeq 0.7$ for one $\mathrm{N} v$ case which meant, that the excitation from the $2^{2} P$ levels to the $3^{2} D$ levels was twice as strong as the total excitation from the 
ground state. This was not sufficient to separate the two excitation rates, so we quoted only the average.

However, when going to higher electron densities in the laboratory, one has to be aware that the resonance lines can become optically thick, which complicates the analysis. In addition, collisional processes between the $n=3$ levels become important. One might also have to take into account cascading from the $4^{2} F$ term, since it decays radiatively only to the $3^{2} D$ levels. This becomes especially important at higher electron densities where the $4^{2} F$ levels are coupled collisionally to the other $n=4$ levels. For these reasons I have only some reservations about the accuracy of $20 \%$ you want to achieve, since I think it is already very difficult to obtain a relative calibration of the vacuum ultraviolet monochromator between $120 \AA$ and $770 \AA$ to that accuracy.

H. Nussbaumer: A difference between distorted wave and close coupling calculations exists for CIII $2 s^{2} 1 S-2 s^{2} 2 p^{1} P^{0}$. Osterbrock did a close coupling calculation including the two configurations $2 s^{2}, 2 s 2 p$; Eissner in his distorted wave method included in addition $2 p^{2}$, the ${ }^{1} S$ level of which couples strongly with $2 s^{2}{ }^{1} S^{0}$. It might well be that in this case the distorted wave method gave better re sults simply because better wave functions were used by including configuration interaction. I calculated that same cross section by using a simple impact parameter approximation combined with oscillator strengths obtained from a multiconfiguration calculation; my result agrees well with Eissners. 